\title{
Blockchain-enabled supply chain: analysis, challenges, and future directions
}

\author{
Sohail Jabbar ${ }^{1,2}$ (D) Huw Lloyd ${ }^{3} \cdot$ Mohammad Hammoudeh $^{1} \cdot$ Bamidele Adebisi $^{4} \cdot$ Umar Raza $^{4}$
}

Published online: 20 November 2020

(c) The Author(s) 2020

\begin{abstract}
Managing the integrity of products and processes in a multi-stakeholder supply chain environment is a significant challenge. Many current solutions suffer from data fragmentation, lack of reliable provenance, and diverse protocol regulations across multiple distributions and processes. Amongst other solutions, Blockchain has emerged as a leading technology, since it provides secure traceability and control, immutability, and trust creation among stakeholders in a low cost IT solution. Although Blockchain is making a significant impact in many areas, there are many impediments to its widespread adoption in supply chains. This article is the first survey of its kind, with detailed analysis of the challenges and future directions in Blockchainenabled supply chains. We review the existing digitalization of the supply chain including the role of GS1 standards and technologies. Current use cases and startups in the field of Blockchain-enabled supply chains are reviewed and presented in tabulated form. Technical and non-technical challenges in the adoption of Blockchain for supply chain applications are critically analyzed, along with the suitability of various consensus algorithms for applications in the supply chain. The tools and technologies in the Blockchain ecosystem are depicted and analyzed. Some key areas as future research directions are also identified which must be addressed to realize mass adoption of Blockchain-based in supply chain traceability. Finally, we propose MOHBSChain, a novel framework for Blockchain-enabled supply chains.
\end{abstract}

Keywords Blockchain $\cdot$ Supply chain $\cdot$ Smart contract $\cdot$ Scalability $\cdot$ Interoperability $\cdot$ Consensus algorithm $\cdot$ GS1 Standards

Sohail Jabbar

s.jabbar@mmu.ac.uk

Huw Lloyd

Huw.Lloyd@mmu.ac.uk

Mohammad Hammoudeh

m.hammoudeh@mmu.ac.uk

Bamidele Adebisi

b.adebisi@mmu.ac.uk

Umar Raza

u.raza@mmu.ac.uk

1 CfACS IoT Lab, Department of Computing and Mathematics, Manchester Metropolitan University, Manchester, England

2 Department of Computational Sciences, The University of Faisalabad, Faisalabad, Pakistan

3 Department of Computing and Mathematics, Manchester Metropolitan University, Manchester, England

4 Department of Engineering, Manchester Metropolitan University, Manchester, England

\section{Introduction}

The supply chain is an interconnection of organizations, activities, resources, people and information for transforming natural resources and raw materials into a finished product for delivery to the end customer. Among major drivers of the transformation of traditional supply chain are hypersegmentation, localization of source and produce of products, Manufacturing 2.0, rising customer expectations and end-to-end visibility to companies, suppliers and customers [1]. These factors triggered large technology organizations to initiate a collaboration impacting the whole process of the supply chain with increased automation in a hybrid architecture [2]. This required a holistic approach to integrating the siloed ordering, purchasing, manufacturing and logistics processes into a centralized platform to improve supply chain reliability, agility and effectiveness.

Various sensing, communication, storage and processing technologies such as the Internet of Things (IoT), 5G, cloud computing, Edge/Fog computing and data science 
have enhanced the digital capabilities in the organizational layers of the supply chain. This digital initiative is instrumental in driving the supply chain system toward a higher level of integration and standardization of processes. Companies such as Uber, Careem, Alibaba, Netflix and Airbnb are benefiting from the current disruptive trend toward digital supply chains. Delivery drones [3], robotic goods handling $[4,5]$, pick and drop autonomous vehicles [6], certificate system, [7], eHealthcare system [8] and vision picking in warehouse operations [9] are some of the technologies enabling the operation and governance of goods handling at different stages of a supply chain. Integration of supply chain silos to create a coherent system from disjoint parts through seamless technological interconnection is key to end-to-end track and trace. Though the words tracking and tracing are sometimes used interchangeably in the literature, tracking implies a less rigorous observation of the working path, while tracing requires more careful monitoring and reproduction of the step by step moves of any process or entity.

Traceability can formally be defined as knowledge of the history and location of an entity, either internal to the organization or external to it through any recorded identification. The process varies from industry to industry and product to product. In manufacturing and processing, it begins at the source of each ingredient and proceeds via transportation to end customers through processing, packing and distribution. In agriculture, farming and fisheries, identification begins with the planting of grain or with the birth of livestock and follows the steps until transportation to the market including growth, use of pesticides and veterinary and nutritional records. Similarly, there are several consistent principles involved in the traceability of the supply chain of retail and food services, including the transportation, distribution, manufacturing and production processes.

Traceability can ensure the integrity of a product through the supply chain, but this is a challenging process to manage. Technological solutions for monitoring and recording the flow of goods include code-carrying and non line-of-sight technologies such as radio frequency identification (RFID). RFID tags are widely used on pallets, cases, packages or individual items, and their use is now an indispensable part of the supply chain. A serialized bar code identifier with a numeric and alpha-numeric code format is a cost-effective technology at both the item and case levels. The data therein can also correlate with production data such as product quality and time to market. For product identification, a wellorganized system is developed and maintained by GS1 $\left({ }^{1}\right)$, a not-for-profit organization set up with the aim of developing standards for improving the visibility, efficiency and

\footnotetext{
${ }^{1}$ https://www.gs1uk.org/
}

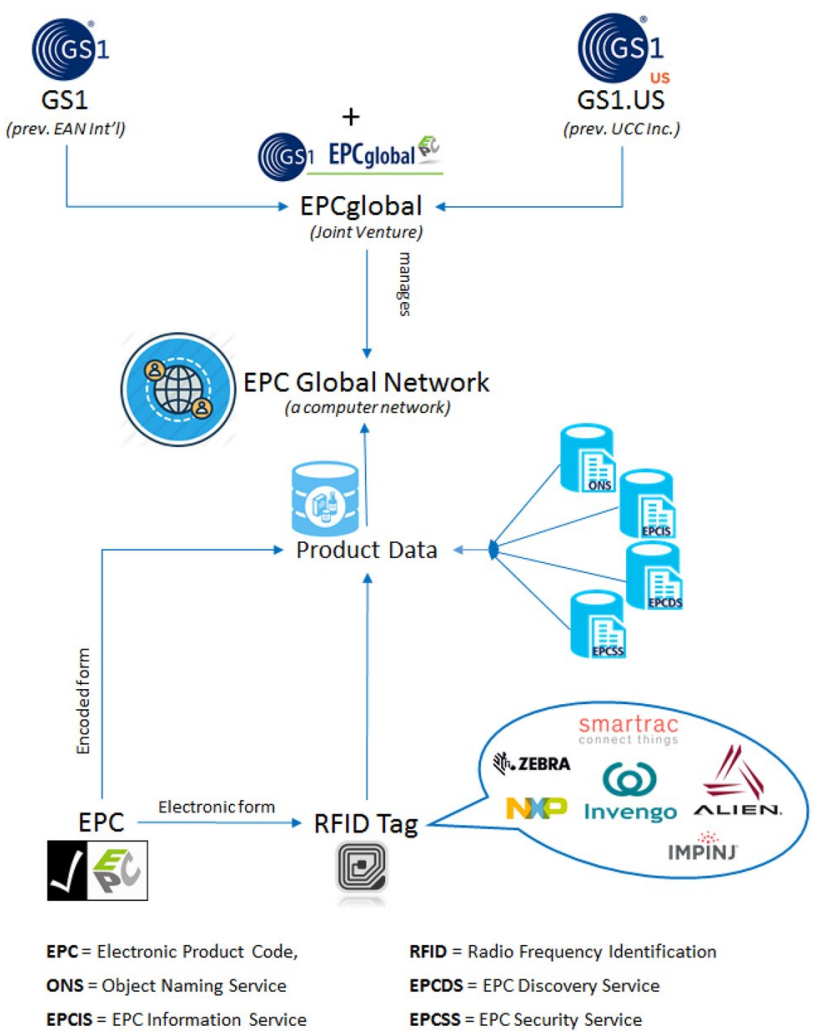

Fig. 1 A depiction of EPCglobal from the data source to EPC Global Computer Network

safety of the supply chain network in both physical or digital aspects.

The services of GS1 can be categorized into three areas: identity, capture and share [10]. GS1's identity standards cover five areas: (i) company and location (Global Location Number - GLN), (ii) product (Global Trade Item NumberGTIN, Serialized Global Trade Item Number-SGTIN), (iii) Logistics and Shipping (Serial Shipping Container CodeSSCC, Global Shipment Identification Number-GSIN), (iv) Asset (Global Individual Asset Identifier-GIAI, Global Returnable Asset Identifier-GARI) and v) Services and More (Global Service Relation Number-GSLN, Global Document Type Identifier-GDTI). G1's capture standards are of two categories: (i) Barcodes Line of Sight, (ii) Electronic Code-Non-line of Sight. The first contains EAN \UPC, GS1-128, ITF-14, Data Bar, QR code, Composite code, and Data matrix. The second contains only EPC (HF\UHF Gen2) for RFID. GS1's share standards have three types of data exchange: (i) event data from a physical or digital object (Electronic Product Code Information Service-EPCIS), (ii) interaction of objects, i.e., transaction data covered by EDI standards (GS1 XML, GS1 EANCom, eCom), (iii) Global Data Synchronization Network (GDSN),an internet-based integration system for sharing business data among partners. This categorization of services of GS1 is tabulated in 
Table 1 Categorization of GS1 services

\begin{tabular}{|c|c|c|}
\hline Category & Standards $\backslash$ covered areas & Technology \\
\hline \multirow[t]{5}{*}{ Identity } & Company and location & - Global location number - GLN \\
\hline & Product & - Global trade item number-GTIN • Serialized global trade item number-SGTIN \\
\hline & Logistics and shipping & $\begin{array}{l}\text { - Serial shipping container code—SSCC } \bullet \text { Global shipment identification number- } \\
\text { GSIN }\end{array}$ \\
\hline & Asset & • Global Individual Asset Identifier - GIAI • Global Returnable asset identifier-GARI \\
\hline & Services and more & • Global Service Relation Number-GSLN • Global document type identifier-GDTI \\
\hline \multirow[t]{3}{*}{ Capture } & Bar codes line of sigh & $\begin{array}{l}\text { • EANBackslashUPC • GS1âĂŞ128•ITFâĂŞ14 • Data Bar • QR code • Composite } \\
\text { code }\end{array}$ \\
\hline & Electronic code-non-line of Sight & - EPC (HFBackshlashUHF Gen2) for RFID \\
\hline & Logistics and shipping & $\begin{array}{l}\text { - Serial shipping container code-SSCC• Global Shipment identification number- } \\
\text { GSIN }\end{array}$ \\
\hline \multirow[t]{3}{*}{ Share } & Event data from a physical or digital object & - Electronic product code information service -âĂŞ EPCIS \\
\hline & $\begin{array}{l}\text { Interaction of objects, i.e., transaction data } \\
\text { covered by EDI standards }\end{array}$ & $\bullet$ GS1 XML• GS1 EANCom • eCom \\
\hline & $\begin{array}{l}\text { Internet-based integration system for shar- } \\
\text { ing business data among partners }\end{array}$ & - Global data synchronization network—GDSN \\
\hline
\end{tabular}

Table 1. The procedural working of EPC in the GS1 system from RFID devices to the capture of various types of product data in a multi-stakeholder environment deployed on the global scale is shown in Fig. 1. Figure 2 depicts the multi-sourced data capturing involving GS1 standards and technologies, synchronization of multi-stakeholders' data and its storage in the GSDN.

Figure 3 depicts the workflow in a typical supply chain, highlighting the incorporation of various smart technologies and standards to facilitate its digitalization. The emergence of digital twins as part of the supply chain analytics is also shown. This analytics process covers visibility into the supply chain, automation of involved processes and integration with other enterprise systems for application base system handling.

\subsection{Contributions}

This survey article is the first to cover a wide range of aspects of Blockchain-enabled supply chain. Here, we summarize our major contributions in this domain.

- Make a comprehensive overview of the digitalization of the supply chain, the role of GS1 standards and technologies, the drawbacks of this existing system and how these are addressed using Blockchain.

- Summarize and analyze a range of state-of-the-art consensus algorithms.

- Critically analyze the major technical and non-technical challenges in the adoption of Blockchain for supply chain applications. An analysis of the core issues of scalability and interoperability is presented, along with available solutions.
- Identify challenges associated with the mass adoption of Blockchain-based supply chain traceability and propose a model, called MOHBSChain, for the adoption of future Blockchain-enabled supply chain.

\subsection{Paper organization}

The remainder of the paper is organized as follows. Section 2 highlights the shortcomings of existing supply chain traceability systems and analyzes the efforts of available solutions to overcome those issues. Section 3 reviews the emergence of the Blockchain-enabled supply chain. To strengthen the claim of emergence, success stories of Blockchain-based supply chain traceability are presented along with a list of real-world use cases. The most successful start-ups in the field Blockchain-enabled supply chain, particularly those focused on traceability, are discussed in this section. Section 4 discusses at length the technical and non-technical challenges to adoption of Blockchain. Among the technical challenges, scalability and interoperability are covered in detail along with the current and proposed solutions. An overview of the most commonly used consensus algorithms is given in Sect. 5. The utilization of consensus algorithms in various supply chains presented in the literature is also given in this section. Section 6 discusses the constituents of the Blockchain ecosystem together with relevant literature covering the topic of application development for the Blockchain-based supply chain. The key research directions arising from the application of Blockchain in a number of domains including supply chain are discussed in Sect. 7. The outcomes of this survey article are presented in Sect. 8. 


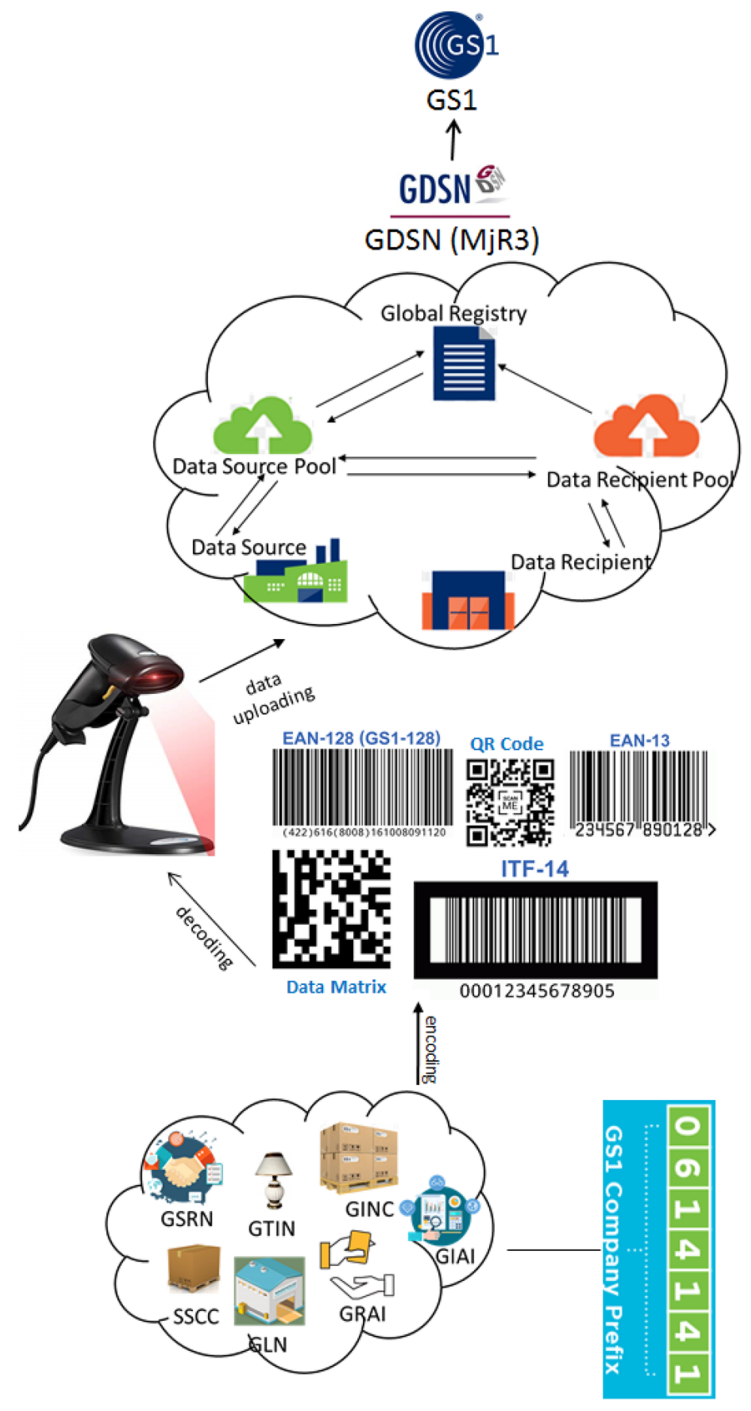

GDSN = Global Data Synchronization Network

MjR3 = Major revision 3

GTIN = Global Trade Item Number

SSCC $=$ Serial Shipment Container Code

GINC = Global Id Number for Consignment

GRAI = Global Returnable Asset Identification

GIA = Global Individual Asset Identifier

GLN = Global Location Number

GSRN = Global Service Relation Number

EAN $=$ European Article Number

Fig. 2 Representation of a complete global data synchronization network from data source to global registry for accessing from multistakeholders of a typical supply chain

\section{Blockchain and smart supply chain}

This section first presents an overview about working of Blockchain along with insight depiction in Fig. 4 and then its application in Supply Chain is given.

\subsection{How Blockchain works?}

The transaction is initiated from the wallet of the node (transaction initiator). All the transactions are first placed

to the unconfirmed transaction pool. The miners pick up the transactions from the pool. Once the transaction is confirmed, SHA256 hashing algorithm is applied on the transaction data (having six parameters) and generates its hash. Similarly, the hash of all confirmed transactions is generated to make up the Merkle tree that ends up at a single hash value called Merkle root. Every confirmed transaction is placed in the miners' own maintained transaction pool. Once the Merkle root is generated, the nodes then solve the puzzle for generating the nonce value. The miner that successfully generates the nonce at first broadcasts its Blockchain having its newly generated block to other network miners. This newly generated Block has the SHA256 algorithm applied to six parameter values including the generated nonce. All other miners stop generating the nonce and work on validating the shared Blockchain by the successful miner. Once the validity of this shared chain is confirmed by more than $51 \%$ miners, it is replicated to all the miners through distributed ledger technology (DLT). The insight of this process is depicted in Fig. 4. Also, the Blockchain protocol stack is depicted in Fig. 5.

To study further on Blockchain and its various aspects including its working, applications, challenges, etc., can be referred to [11].

\subsection{Blockchain in smart supply chain}

Problems in existing supply chain traceability systems, which are either difficult or impossible to solve with current technologies, include establishing reliable provenance, and preventing fraud and counterfeiting. Existing traceability systems adopt either a centralized or distributed architecture [12]. Centralized architectures are managed by an authoritative third party body with the risk of (i) single node attack, (ii) data tampering, and (iii) information disclosure. Distributed architecture, such as an EPCIS based setup, allows for scalability due to the ease of creation and sharing of visibility event data on digital and physical objects, both within and across enterprises. Nevertheless, data tampering and information disclosure issues remain unsolved in EPCIS-based systems [13]. Moreover, the majority of current IoT solutions still rely on heavily centralized cloud infrastructure, which results in lack of transparency and by nature faces security threats, including availability, auditability, data lock-in, and confidentiality [14]. Blockchain is a promising technology for addressing these issues which brings three major benefits: (i) secure traceability and control, (ii) data immutability and (iii) trust creation, in relatively low cost IT solutions.

Blockchain-based systems are an amalgamation of cryptography, public key infrastructure and economic modeling, applied to peer-to-peer networking and decentralized consensus to achieve distributed database synchronization [15, 


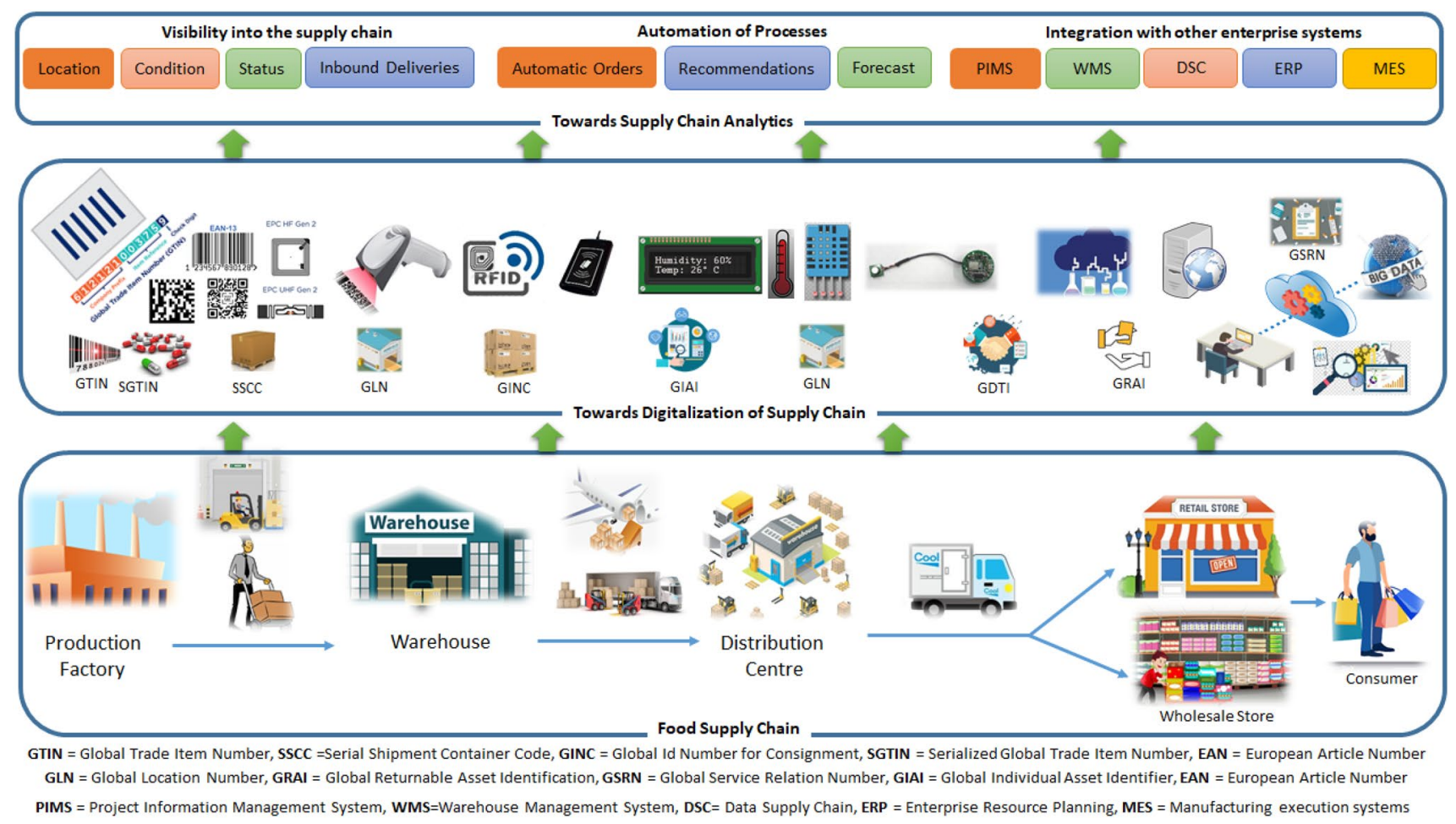

Fig. 3 Transition from traditional to digitized supply chain

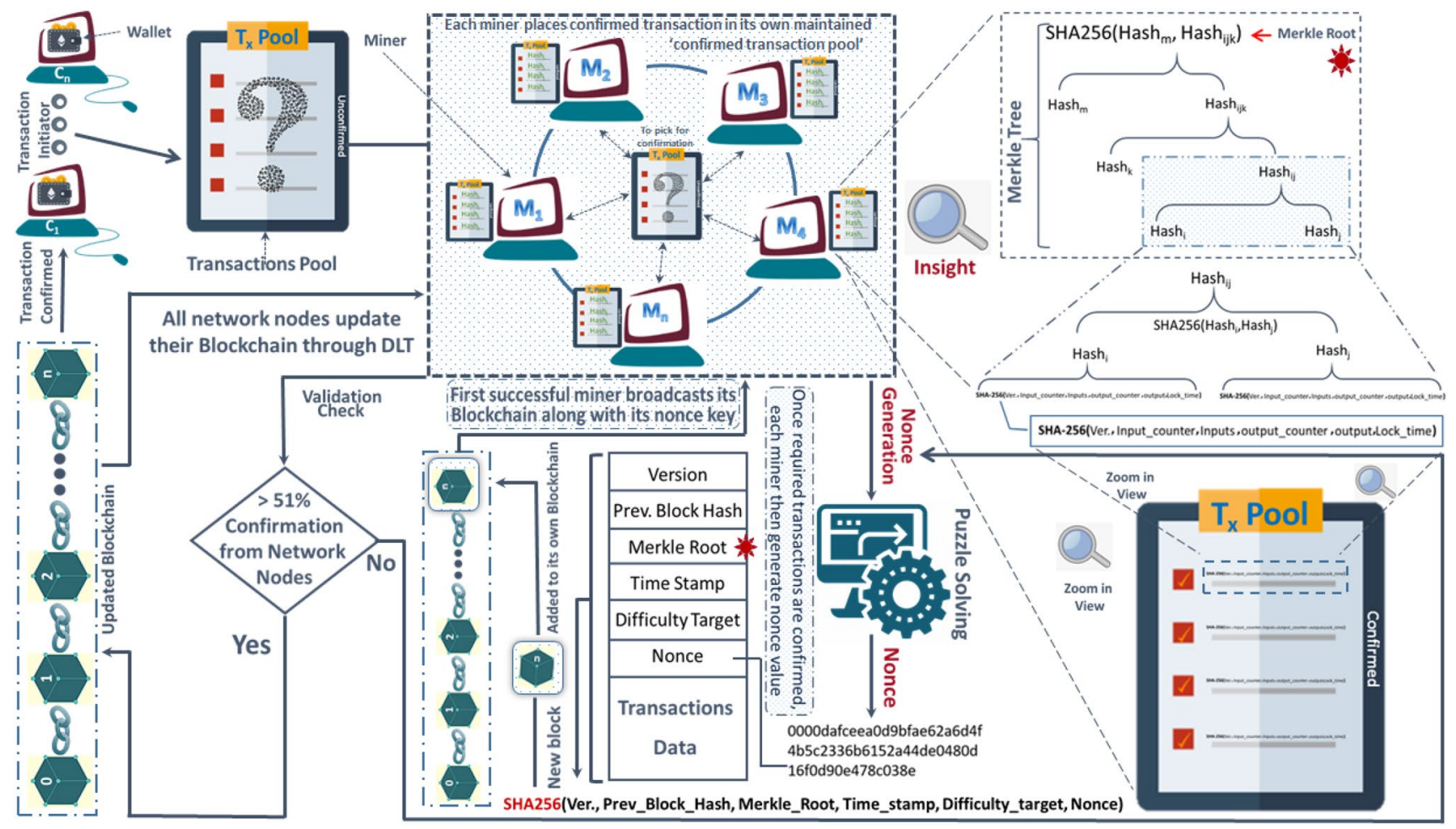

Fig. 4 How Blockchain works? - insight depiction 
Low Power loT Devices

\section{Single Board Computers}

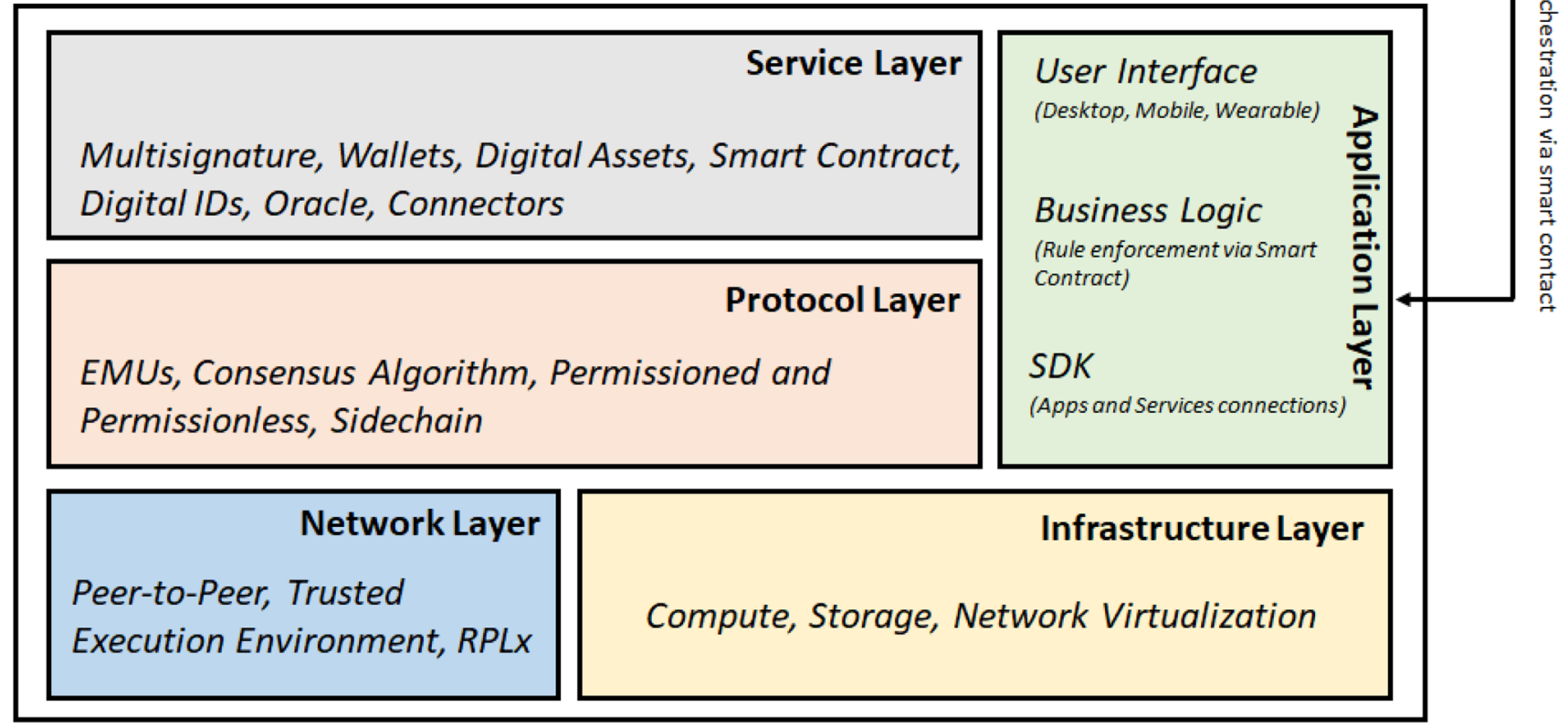

Fig. 5 An overview of the Blockchain protocol stack

16]. It is a revolutionary technology which promises major changes in the operation of global technology. It has been adopted in various industries and domains including supply chain [17], monetary-use cases [18], asset management $[19,20]$, land-record registry traceability[21], vehicular network [22], e-contracts [23], retail [24], decentralized exchanges [25], business modeling [26], energy trading and sharing [27], transaction process automation [28], and mission critical scenarios [29]. Opinions on Blockchain among technologists, practitioners and other industry stakeholders are divided; both proponents and opponents have their standpoints.

There are a number of arguments against Blockchain. Its distributed nature requires it to carry out the the same processes as a traditional database, as well as additional tasks. These are signature verification, attaining consensus, and redundancy in nodes' processing due to the large network size. These processes may adversely affect the performance in several areas such as consumption of energy, time and other resources. The double spend attack, also known as the $51 \%$ attack, is a serious security flaw in the Blockchain [30]. Though the Bitcoin platform has proven to be very efficient and resilient over the years with no evidence of a successful $51 \%$ attack, in smaller Blockchains there is an increased chance of attack, as seen in Bitcoin Gold, Krypton, Shift and others [31]. The immutability of data in the Blockchain requires that a hard fork is performed to rectify errors; the trustworthiness of data input into the Blockchain (by human or machine actors) must therefore be ensured, which is a significant challenge when using Blockchain as a distributed data store. Other concerns include limited scalability and storage [32], and uncertain regulatory status.

\section{Emergence of Blockchain-enabled supply chain}

Blockchain is beginning to be adopted in various supply chain applications, especially in the financial and manufacturing sectors, for optimizing processes and improving efficiencies in other aspects. The disruptive presence and integration of Blockchain with IoT have made it one of the most promising recent technological trends. This combination of technologies is allowing companies to strengthen relationships among their core business stakeholders, especially with current customers, and to attract the new ones. As an example, it is estimated that the businesses operating in the food and pharmaceutical industries are facing big 
financial loses to a variety of supply chain issues. These include counterfeiting, stolen products, gray market, fraud and product recalls. Such factors have prompted a movement of supply chain stakeholders toward more transparency and traceability. As Blockchain promises immutability, transparency, security and fault tolerance, it is a promising solution, at least for the trust and traceability issues. In practice, there are already companies that use this technology to support the process, and some have already shown promising results. Walmart in collaboration with IBM has successfully launched a pilot project for the traceability of mangoes from the farm to fork [33]. Walmart, IBM and Beijing Tsinghua University collaboratively launched a project to build a model using Blockchain technology for tracking and tracing in the pork industry in China [34]. IBM's partnership with Walmart is one among nine other initiatives providing Blockchain-enabled food supply chains as part of the IBM Food Trust project. Walmart has already announced to its leafy green vegetable suppliers that they are required to upload their data to the Blockchain by September 2019. The latest update on bringing the unprecedented transparency by Wallmart to the food supply chain can be found at [35].

Table 2 shows examples of real-world use cases of Blockchain-enabled supply chain for various purposes, particularly for traceability.

Another successful Blockchain-based solution to track the provenance of high-value assets is Everledger [45]. More than two million diamonds have been uploaded to the Blockchain for complete provenance from origin to the end owner. This history tracking is achieved using pictures or certificates at each step of the way. Carrefour, Europe's largest retailer, provides shoppers with the provenance of free-range chickens in Auvergne, France, by integrating their system with Blockchain food traceability [46]. In this project, Carrefour has expanded the use of Blockchain to other food items including salmon, tomatoes, honey, eggs, and milk. Nestlé, a Swiss food company, and British-Dutch Unilever are also participating in Blockchain projects to improve food supply chain traceability.

The smart contract is a feature of Ethereum Blockchain which enables organizations to handle large amounts of transactions automatically with full confidence for direct and indirect stakeholders. Even in some Fortune 100 companies, supply chains can include sales outstanding of up to 60 days, despite a typical 30-days credit contract limit [47]. In these cases, management of outstanding invoices is a challenging problem. Blockchain-powered smart contracts are now enabling this process to be automated with programmable clauses. Once a set of conditions is satisfied, the contract is triggered to run automatically, thus speeding up the sales cycle.

The process of moving goods across borders is heavily regulated; the clearance procedure is mainly processed manually, adding up 15 to $50 \%$ of the total shipping costs. For example, to ship refrigerated products from East Africa to Europe, one would have to obtain written approvals from nearly 30 organizations. Maersk partnered with IBM has already initiated a transformation of their business model using Blockchain-powered smart contracts with the name TradeLense [48]. Their first pilot project built on top of legacy systems is in operation since 2017. This system services a large network of shippers and their daily operations and documentation in a bid to lower the shipping costs. The National Livestock Identification System (NLIS) is AustraliaâĂŹs world-leading system for the identification and traceability of livestock. It tracks livestock through the entire process from birth to slaughter. A report conducted by Meat and Livestock Australia [49] evaluates the use of Blockchain technology to support provenance. It looked into the utilization of Blockchain by different participants in the supply chain to satisfy the provenance life cycle. They concluded with an intention toward its practical implementation in their system. Similarly, the integration of the Beef Infoxchange System (BIXS) with the Canadian Livestock Traceability System (CLTS) has taken positive steps towards the implementation of a Blockchain-based traceability system [50]. Canada enforces the use of bar codes, plastic ear tags, or two electronic button ear tags to identify the initial herd.

These successful projects have motivated other market players to adopt Blockchain-based solutions. Based on thorough and extensive research on the potential of Blockchain in the supply chain industry conducted by a team of innovation analysts at Innovator's Guide, there are around 800 startups around the globe working in the field [51]. Table 3 presents some of the most successful startups of Blockchainenabled supply chain having diverse focus, particularly on traceability.

\section{Blockchain adoption challenges in supply chain applications}

Although the technology itself is revolutionary, there are many limitations and challenges in its adoption in applications. Considering the length of the article, a short but comprehensive discussion is made on non-technical and technical challenges Blockchain Adoption in Supply Chain.

\subsection{Non-technical challenges}

The availability of technology does not guarantee its uptake; even now, many warehouses still operate with paper at the integral points, although RFID chips and scanners are now conveniently available, in reach and access. Factors acting against the adoption of technology include the lack of 


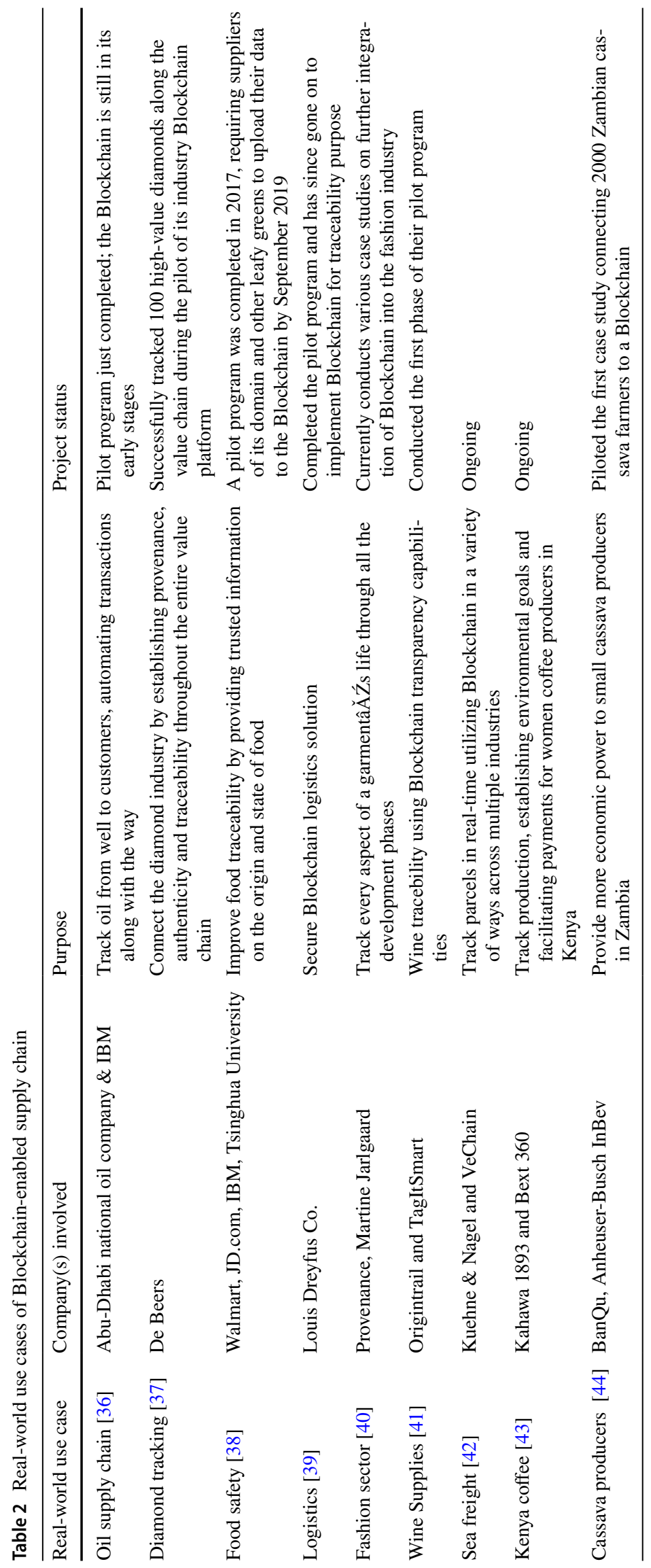


Table 3 List of some successful startups of Blockchain-enabled supply chain applications

\begin{tabular}{|c|c|c|c|c|}
\hline Name & Location & URL & Year & Business focus \\
\hline DOR $Е$ & UK & dorae.com & 2014 & Digitize \& automate document processes \\
\hline ShipChain & USA & shipchain.io & 2017 & End to end supply chain solution \\
\hline Zego & USA & zegofoods.com & - & Provide traceability to food products $\&$ ingredients \\
\hline Provenance & USA & provenance.org & 2014 & $\begin{array}{l}\text { Empower brands to communicate the origin and impact of their } \\
\text { products }\end{array}$ \\
\hline Eximchain & USA & eximchain.com & 2015 & $\begin{array}{l}\text { Reduce procurement cost \& paperwork, and increase order fullfill- } \\
\text { ment \& transparency }\end{array}$ \\
\hline OriginTrail & Hong Kong & origintrail.io & 2013 & $\begin{array}{l}\text { Enabe a universal, collaborative } \& \text { trusted data exchange for inter- } \\
\text { connected supply chains }\end{array}$ \\
\hline PeerLedger & Canada & peerledger.com & 2016 & $\begin{array}{l}\text { Protect human rights, reduce safety risks \& improve environmental } \\
\text { performance in their supply chains }\end{array}$ \\
\hline Blockhead Technologies & Canada & blockheadtechnologies.com & 2017 & $\begin{array}{l}\text { Increase product traceability \& improve data governance for supply } \\
\text { chain organisations }\end{array}$ \\
\hline Zero1 & Spain & zerolcapital.com/ & 2017 & Provide supply chain financing early in the production cycle \\
\hline Cargocoin & UK & thecargocoin.com & 2017 & $\begin{array}{l}\text { Link the physical worlds of trade, transport and logistics with } \\
\text { Blockchain }\end{array}$ \\
\hline Tradeline & Cyprus & tradeline.io & 2017 & Provide post-trade workflow automation platform \\
\hline TangoTrade & USA & tangotrade.com & 2018 & Offer payment assurance to suppliers \\
\hline Hijro & USA & hijro.com & 2014 & Connect financial \& supply chain systems \\
\hline Modum & Switzerland & modum.io & 2016 & Monitor, automate \& optimize supply chain \\
\hline Chronicled & USA & chronicled.com & 2014 & Help supply chain companies create standards \& best practices \\
\hline Sweetbridge & UK/USA & sweetbridge.com & 2016 & Keep all parties involved in a transaction in synchronisation \\
\hline Skuchain & USA & skuchain.com & 2014 & $\begin{array}{l}\text { Incentivize collaboration without compromising security or intel- } \\
\text { lectual property }\end{array}$ \\
\hline WAVE & USA & wavebl.com & 2014 & Securely store and exchange logistics documents \\
\hline Blockverify & UK & blockverify.io & - & $\begin{array}{l}\text { Anti-counterfeit measures of products, goods, merchandise \& } \\
\text { transactions }\end{array}$ \\
\hline Fr8 Network & USA & fr8.network & 2017 & $\begin{array}{l}\text { Improve operational efficiencies, compliance monitoring \& payment } \\
\text { settlement }\end{array}$ \\
\hline
\end{tabular}

understanding of Blockchain among business leaders, the view that it is a fad, and waiting for wider adoption before committing. Even those business leaders who realize the potential of Blockchain are hesitant to invest money and time into it, keeping in view the lack of industry-wide standards and practices. Hence, for Blockchain to be successful, everyone in a typical supply chain industry needs to be convinced of its benefits; the key stakeholders should be on-board and be able to see the benefits in introducing Blockchain. Hence, market acceptability is a key challenge to face.

There is also a lack of enterprise resource planning (ERP) tools and support within existing systems. Typical ERP systems used in many organizations do not support Blockchain. Hence, a bold step is required to either outsource the application development for its particular supply chain or set up in-house development. In the former case, apart from other risks, privacy leakage can be a major concern, as an organization has to hire the services of the third party who must be trusted with the organization's data. In the latter case, privacy risks are lower, but significant investment is required for the long term. Either existing staff require training or existing professionals with these skills must be employed. This may be difficult, since the development requires a range of software skills, as well as requiring an understanding of the economic and business issues and of the underlying supply chain setup.

Though there are other non-technical challenges in addition to those described above, they are mostly variants or extensions of these. Similar lists are available in the literature in $[52,53]$.

At the boundary of the technical and non-technical challenges is the issue of correctness of entered data. Data entered to a Blockchain must be correct; the immutable and transparent attribute of Blockchain technology means the user cannot easily update or modify the entered record. If a supply chain partner is using an unreliable system to record information, then the addition of Blockchain technology can become more detrimental rather than facilitating the user. The immutability of the Blockchain does not guarantee the quality of the data. 


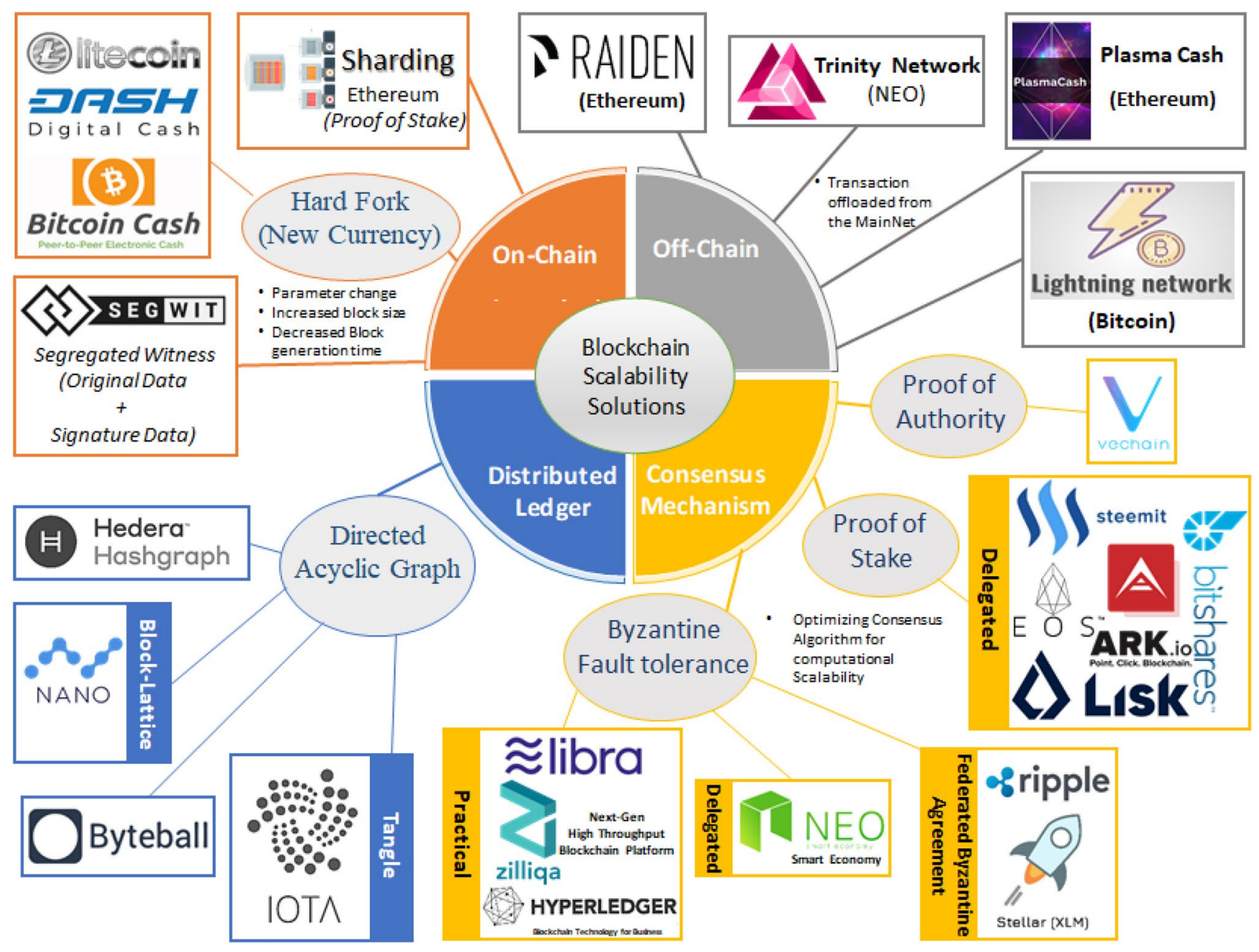

Fig. 6 A taxonomy of Blockchain scalability solutions

\subsection{Technical challenges}

Compared to a traditional database, Blockchain is significantly slower in retrieving and committing records. It also requires significantly more computing resources, and the scalability of these resources is a significant concern. Moreover, interoperability between all systems interacting with the Blockchain is needed. The payment term must be short and flexible enough to be able to cash in any other currency (including FIAT money). In the preceding subsections, we focus on these two issues in the following subsections.

\subsubsection{Scalability}

Scalability is the ability of a system to continue to respond and function after increasing the size of the input to fulfill user demand. To address the issues relating to scalability, Soohyeong Kim et al. [54] categorize the scalability methods into on-chain, off-chain, side-chain, child-chain, and inter-chain solutions, while in [55], Junfeng Xie et al. grouped the scalability solutions into technologies related to the number of transactions, block interval time, data storage and data transmission. We present a categorization of scalability solutions into four types; (i) on-chain scalability (ii) off-chain scalability (iii) consensus mechanism-based scalability (iv) distributed acyclic graphs based scalability. Figure 6 depicts these categories of scalability solutions with a presentation of particular solutions under these categories.

- On-chain solutions require a structural or fundamental change to Blockchain, and so the modification in the underlying rules of the protocol. This is technically known as a hard fork or controversial hard fork in cases where there is a split in the community, since there is a formation of groups that accept or reject the proposed update. Sharding [56], SEGWIT [55] are major solutions. From the hard fork solutions category, there are litecoin, DASH, and Bitcoin Cash, to name a few.

- The off-chain approach uses secondary protocols built on top of the main Blockchain and hence is referred to as 


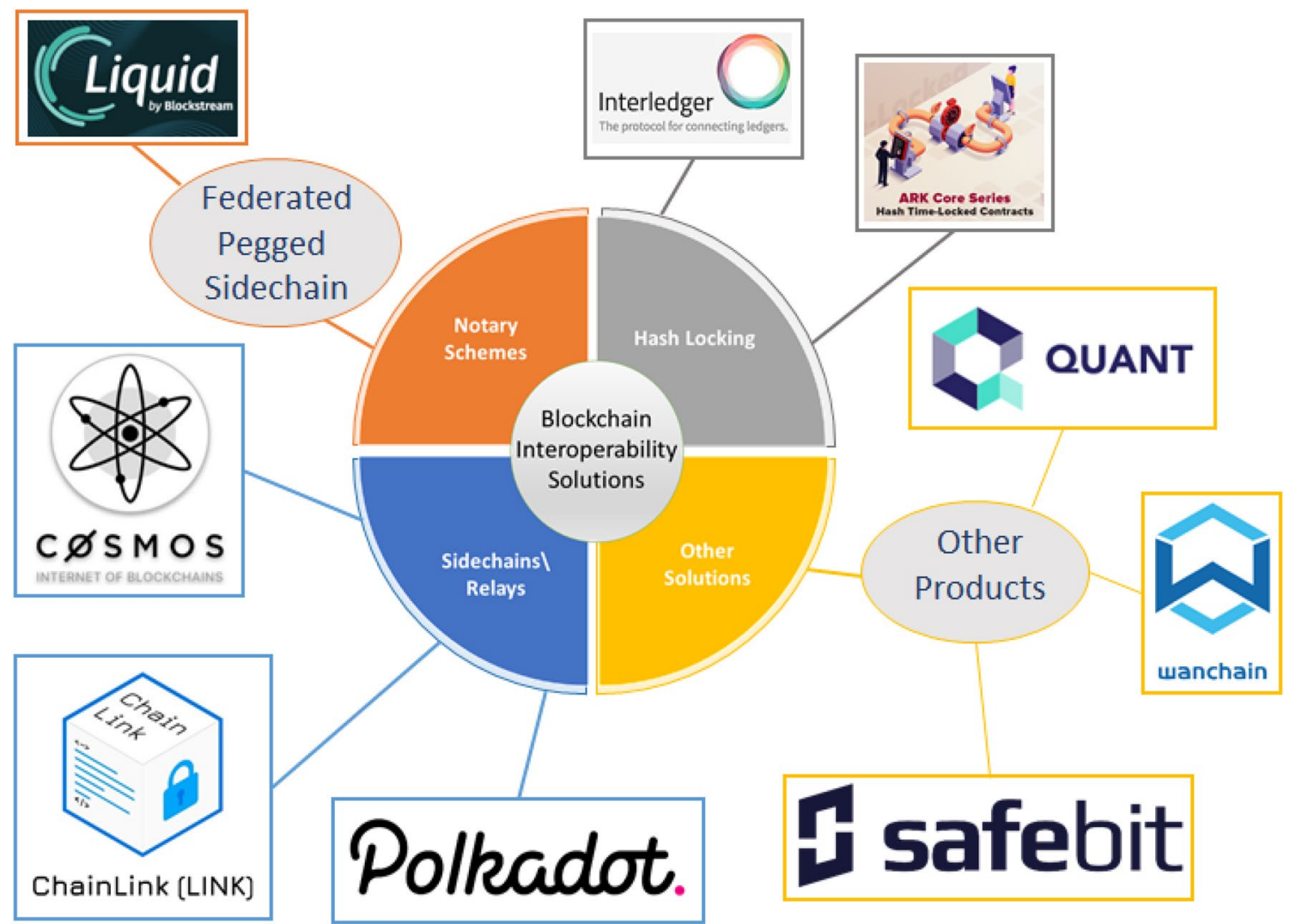

Fig. 7 A summary of Blockchain interoperability solutions in the literature

a second layer scalability solution [57]. In this approach, transactions are off-loaded from the main Blockchain and performed privately between the interacting partners. It confers the advantages of reduced congestion in the MainNet, increased throughput, reduced transaction fees and space saving. Among the available off-chain solutions, some are RAIDEN, Trinity Network, Plasma Cash [58], and Lightning Network [57].

- In Consensus Mechanism based Scalability, the working of the consensus algorithm is optimized to aid in addressing issues of scalability [59]. VeChain using Proof of Authority, ARK.io, LISK, bitshares, E.O.S, and steemit using Delegated Proof of Stake, Ripple, and Stellar using Federated Byzantine Agreement, NEO using Delegated Byzantine Fault Tolerance, Libra, zilliqa, and Hyperledger are major solutions in this category.

- Distributed Acyclic Graph-Based Scalability is distinct from Blockchain. It is another prominent form of distributed ledger [60] technology. Transactions operate independently and asynchronously without conforming to a particular process. The system uses a topological ordering data structure for maintaining transaction records. DAG distributed ledger technology does not suffer from the scalabilty issues as is inherent in Blockchain. Some of the solutions in this category are IOTA, Byteball, $N A N O$, and Hashgraph.

\subsubsection{Interoperability}

Though the adoption of Blockchain is increasing, the isolation of Blockchains in their respective 'silos' due to the lack of interoperability standards is an impediment to wider adoption. Resolving collaboration and cross-chain interaction issues among public, private, and consortium Blockchains can pave the way for a hyper-connected world. Blockchain systems need to speak the same language, and to incorporate and share common capabilities and feature sets related to consensus models, transaction, and contract functionalities. The proposed solutions for Blockchain interoperability can be grouped into three categories, namely (i) Notary Schemes [61], (ii) SideChain \relays [62], and (iii) Hash Locking [63]. Figure 7 summarizes these solutions, giving a visual overview of this section.

- In Notary Schemes, an intermediary trusted entity called a notary witnesses and confirms the state of the interacting Blockchains to enable operations. Liquid using 
Federated Pegged Sidechain a major solution in this category.

- Relays scheme is used many interoperability solutions. Cosmos, Polkadot, and ChainLink are names of a few.

- Hash Locking is the most practical approach to the Blockchain interoperability, although it is limited in functionality. Interledger Protocol (ILP) [64] and ARK Core Series are its some available key solutions.

\section{Consensus algorithms for supply chain applications}

The Consensus Algorithm (CA) plays an indispensable and inseparable role in Blockchain technology. The availability of a rich list of consensus algorithms reflects the engagement of a good number of academicians, researchers, and industrialists in making their contribution toward the emergence of Blockchain. Each has its pros and cons, and any of these singly does not fit all situations, scenarios, and applications. An abstract collective overview of the most renowned CAs is in proceeded paragraphs.

Proof of Work (PoW) carries out large, but completed computation with no further need for any additional work for proof check. The heavy and truthful chain among the competing chains is finalized. PoW limits the rates of new blocks, and it is expensive to add an invalid block as well. A variant of PoW is GHOST consensus algorithm. It is based on the Greedy Heaviest-Observed Sub-tree strategy. Instead of the longest chain consensus rule, the technique here is to follow the path of the sub-tree with the combined hardest proof of work. Proof of Stake remedies PoW by eliminating heavy computation and higher energy consumption as the block creation is tied to the amount of stake. In $B F T$, the new block is only accepted once a voting process among the nodes with the selection of trusted validators is completed. Hence trusted entities work together to add records. Stellar Consensus Protocol(SCP) is a mix of PoW and BFT. The former is used for identity management, while the latter is for the agreement process. A further extension to SCP is $P i$ Consensus algorithm that is specially designed for the low energy \computational power devices such as home PCs and mobile phones. It is possibly slower than SCP, but far faster than PoW. SCP also has its role in eliminating the heavy communication and other resources overheads.CheapBF (trusted hardware) and XFT [65] are from the same family of BFT and give similar advantages as SCP. Many variants of BFT are there in the literature with their pros and cons: HoneyBadgeBFT (Asynchronous BFT protocol) [66], Lin$B F T$ (Linear-Communication protocol for the public chain) [67], Democratic BFT (solves the consensus deterministically with multiple proposers) [68], Aleph (treated the BFT protocol as composition of instances of abstractions) [69],
BFTRaft (a mix of original Raft algorithm and PBFT algorithm) [70] and many more. BitCoin-NG consensus algorithm [71] decreases the latency and increases the throughput through its technique of leader election to append the micro-blocks.

Sylim Partick et al. [72] developed a distributed application engaging smart contract and Swarm as a Distributed File System. In their proposal, they proposed to modify the Prof-of-Work consensus algorithm of Ethereum into a Delegated PoS of PBFT by considering their better scalability feature and also aligning it well with the drug supply chain environment. They proposed to engage the FDA, manufacturer, wholesaler, retailer, and the consumer portal as simulation nodes for running the consensus algorithm for their particular Blockchain. Mao Dianhui et al. [73] designed a novel Food Trading System with CONsortium Blockchain (FTSCON). The target is to establish a sustainable and credible trading environment by eliminating the information asymmetry in the food trade. To improve efficiency, the new consensus algorithm is designed based on Practical Byzantine Fault Tolerance. Its underlying principle is using the automatic transaction mechanism of the system to verify the transaction information. Huang Yan et al. [74] proposed a scenario-oriented Blockchain system for drug traceability and regulation called Drugledger. To ensure the authenticity and privacy of traceability data, they separated the service provider into three independent service components in their reconstructed service architecture. The communication module uses gossip to implement a p2p network. Algorand cryptocurrency is exploited for its algorithm to implement the consensus module. Users in Drugledger are weighed on the number of valid transactions in the past, whereas Algorand weights its user are weighed based on the owned balance.

\section{Blockchain based application development}

The majority of Blockchain-based supply chain systems are based on Ethereum or its variants due to its support for smart contracts. It also has a well-established support and development community. In this section, we discuss a number of recent Blockchain applications, with particular reference to the tools and technologies underlying the implementation.

Kuhn Marlene et al. [75] proposed a consortium Blockchain based on Ethereum or Hyperledger for securing the process quality in manufacturing chains in autonomous driving. Westerkamp Martin et al. [76] designed a supply chain traceability system that models manufacturing processes as token recipes. The system is a prototypical implementation for the Ethereum Virtual Machine using smart contracts.

Khalid et al. [77] addressed the track and trace across the agricultural supply chain with an approach that leverages 
the Ethereum Blockchain and smart contracts. Their consensus algorithm eliminates the need for a trusted centralized authority and intermediaries. It performs efficiently and allows business transactions among all participants within the supply chain ecosystem. All transactions are recorded and stored in the Blockchain with links to a decentralized inter-planetary file system.

Lin Qijun et al. [13] proposed a food safety traceability system based on Blockchain and EPC information services. The prototype system was implemented on the Ethereum Blockchain. For on-chain and off-chain data, they further propose a management architecture that alleviates the data explosion issue of the Blockchain for the IoT. For trusted interaction among the participants, they designed an enterprise-level smart contract. This is tested to prevent data tampering and sensitive information disclosure. MongoDB V.3.6 is used to store and manage food event data and a JSON query is used to interrogate the objects and arrays embedded in the documents. This application used the Ethereum Geth 1.8.2 Blockchain module in JAVA 8.0.1610.12 under Windows 7.

In [73], the authors used Geth v1.6.4 10 for all of the empirical evaluations. The system including the Blockchain consortium, web server and the client browser was designed by using the Ethereum architecture. The web server was developed in PHP, and HTML/CSS/JavaScript was used for writing the client browser page.

Baralla Gavina et al. [78] implemented the from-farm-tofork (F2F) model currently in use in the European Union, which can integrate current traceability rules and processes. The access to the system is only issued to members recognized as legitimate participants in the process. Hyperledger Sawtooth is used as the underlying Blockchain architecture and applications are developed in Python 3. The application subsystem is composed of a web client, a mobile client, an off-chain repository and a set of REST APIs which facilitate the creation and forwarding of transactions and batches to the sawtooth network nodes.

Helo Petri and Shamsuzzoha Ahm [79] proposed Afor, which is built on top of an Ethereum Virtual Machine (EVM) within the Blockchain architecture to operate as a run time environment for smart contracts. The Blockchain application (Tracker App) communicates with EVM and the network. Ethereum node components are called for the implementation of the tracker App. The web applications are developed using HTML, CSS and JavaScript, and Solidity smart contract is used for back end development.

Knirsch F, et al. [80] introduced the implementation of a fully custom, private, and permissioned Blockchain from scratch. They cover the hardware aspects of a system that comprises three core components; nodes, clearing server, and asmartphone app. Xu X, et al. [81] proposed a Blockchain-based traceability system, originChain, and gave detailed working on its design. A Blockchain development ecosystem comprises the choice of Digital Ledger Technology (DLT) type, DLT accessibility scope, Blockchain platform and CA. Supporting development tools include Integrated Development Environments (IDE), libraries, testing tools, programming languages for smart contract and application development, and storage applications. The composition of this ecosystem strongly depends on the application area which is targeted. In the remainder of this section, we give a brief introduction of these constituents, which are also summarized in Fig. 8.

- DLT-A digital database system for recording the transaction of assets that is consensually shared and synchronized across multiple sites or geographies, and having public witnesses. It can either be Blockchain based or non-Blockchain based.

- Accessibility scope-Users interacting with the Blockchain may or may require permission to access the system. Permissioned or private Blockchain requires the participants or validators to be invited by an administrator to access the system, while non-permissioned or public Blockchain does not have this requirement. A mix of both is a federated or consortium Blockchain.

- Blockchain platform-A long list of Blockchain platforms or frameworks is available, with varying suitability for a particular scenario. A list of some of the most notable frameworks is presented in Figure 8.

- $C A-$ This is the procedure through which the peer nodes reach a common agreement for validating the current state of the distributed ledger. It maintains the integrity and security of every Blockchain and achieves reliability in the network. The acceptability of any consensus algorithm to the Blockchain is dictated by the application requirements. Figure 8 shows the previously discussed names of consensus algorithms.

- Integrated Development Environment-Refers to a suite that consolidates the basic tools required to write and test the software. Some of the most popular IDEs and editors for the Blockchain application development are also shown in Figure 8.

- Development testing tools and libraries-Extending the IDE, development and testing tools and libraries provide support for development of applications which interact with the Blockchain.

- Programming languages-Blockchain development uses a wide variety of programming languages including some specific to Blockchain development. The selection criteria may consider the requirements for security, resource management, performance, and the need for isolation level.

- Storage applications - The storage of transaction-related data could be on-chain or off-chain depending upon its 


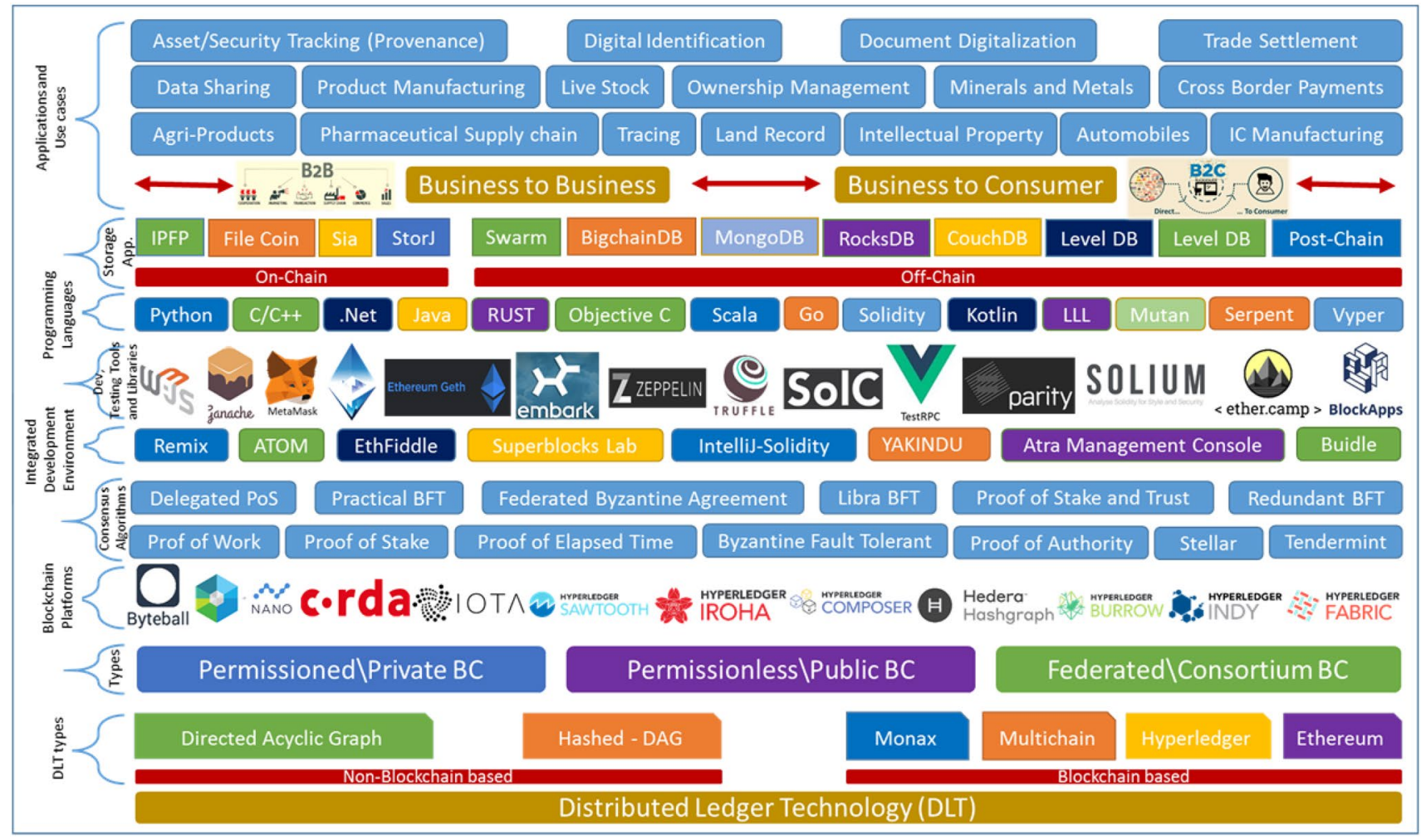

Fig. 8 Blockchain ecosystem-A Presentation covering types of DLT, key constituents of Blockchain application development environment, and Blockchain applications and use cases

sensitivity level or its volume. Most of the off-chain storage applications are the same as are previously used with big data.

- Applications and use cases-The two major categories of Blockchain-based applications are business-to-business and business-to-consumer. A list of applications and use cases of Blockchain are presented in the same figure.

\section{Research directions}

The adoption of Blockchain in complex applications such as supply chain exposed many challenges and open research problems. The following list identifies some key areas which must be addressed by researchers to realize mass adoption of Blockchain in supply chain traceability.

- Built-in analytics for Blockchain- At present, Blockchain data is purely a transaction repository without any access to the data-parallel processing systems such as Dryad Spark, Flink or MapReduce. It is required to scan the Blockchain data before running analytics. Implementation of the input reader with the execution engine could assist in developing built-in analytics for the Blockchain. Edge Analytics capabilities could also be introduced.
How this integration with current Blockchain systems to enable built-in anlytics can be achieved is an open research area [82, 83].

- Integration and analytics across on-chain and off-chain data-As an extension to the previous challenge, analytics could span across on-chain and off-chain data systems. Federated search, query processing over federated data, i.e., query federation, are the emergent research areas in the integration and analytics across on-chain and off-chain data. Furthermore, optimal checks on the strategies that export on-chain data to the off-chain database and where analytics are applied need to be explored and developed. Another challenge is the maintenance of immutability in the exported data and data security in the context of analytics over both on-chain and off-chain data [84].

- Development of smart contract templates-The programmed logic of Blockchain smart contracts is usually manually developed after studying related documents. This methodology is not only time consuming, but also error prone. There is a need to define a standardized semantic framework for the smart contracts to make it a fully automated process. Another related research challenge is to develop standardized templates of smart contacts for various legal issues and assignments. This 
automated template development requires mature natural language processing algorithms to extract related data and operational parameters from legal documents, and embed this in smart contract templates [85].

- Development of security analysis technologies for smart contracts - In the event of a smart contracts becoming compromised, the programmed logic and the Blockchain data are exposed, with potentially disastrous consequences, and backdoor attacks may be used to exfiltrate data from the Blockchain. This requires the development of strong security analysis technologies for smart contracts. There is also a need to develop techniques for assessing the semantic trustworthiness of smart contracts between the interacting parties. Other related open questions including detecting bugs in smart contacts, dealing with wrongly behaved smart contracts, and updating them with correct code without impacting the running network require research attention. Encapsulating all the developed security analyses for the smart contracts with the confidentiality, security and privacy assurances at different levels is needed for compliance with regulatory bodies and instruments such as HIPAA and GDPR [86, 87].

- Standardization and automation of governance-The application of Blockchain smart contracts in governance and services could allow for the transformation of government roles and functions. This may in turn impact transparency of governance and services, establishment of smart and trustworthy government, flattening of organizational structures, and improved security of government data. Despite these potential benefits, Blockchain technology also poses many challenges to government in the development of appropriate laws, regulations, and management mechanisms. A key issue here is that the central philosophy behind Blockchain technology is at odds with prevailing paradigms of government: Blockchain is a decentralized system with no third-party intervention, whereas government classically relies on centralized authority. This is a societal and political, rather than technological challenge $[88,89]$.

- Query execution system for encrypted data in Blockchain-Considering the complex features of Blockchain, most of the data access technologies and best practices from previous technology movements result impractical to it. The Web 3.0 deserves a Web 3.0 data access protocol. Decentralization, opacity and sequential data storage are major causes of this challenge. There is demand of query execution system for the encrypted data in Blockchain [90].

- Trusted electronic data management systems-verification mechanisms for the correctness of entered dataThe immutability feature of Blockchain gives rise to challenges as well as benefits. The challenge arises from the need for data entered to the system to be strictly correct when first entered, with no opportunity for correction of errors. Devising verification mechanisms for the correctness of data entered to the Blockchain transaction repository is an active area of research, in which trusted electronic data management systems are a key focus. A related challenge is the security of attached data-generating technologies such as IoT, RFID and sensors. Though much research work has been carried out, there is a need to reconsider the solutions from the perspective of these technologies integrated with Blockchain.

- Interoperability standards-For organizations to migrate existing systems toward Blockchain with confidence, defining interoperability standards is of core value [91].

- Improving scalability in throughput, storage, and network communication-With the increase in integration of Blockchain with existing systems, the scale of data, data processing and manipulating requirements and importance of timely finalizing of transactions are increasingly important issues. There remains a large gap between the requirements and the available solutions for improving the Blockchain scalability [92].

- Incorporation of right-to-forget mechanism in Blockchain-The retroactive erasure of personal data from all distributed copies upon request is a statutory requirement in some jurisdictions, seemingly in direct conflict with the core immutability feature of Blockchain. This rightto-forget or 'Right-to-be-Forgotten' is a requirement imposed by the GDPR in European Union Law. Blockchain compliance with the regulation is challenging, if not impracticable, since it requires Blockchain data be editable or deleted on requested. Though some efforts on addressing these regulatory requirements while preserving Blockchain security are available in the literature, there is some way to go and this is an open research question $[93,94]$.

To support the implementation of Blockchain in the supply chain, we propose a model which combines the ideas presented in this paper into a coherent model for a Blockchain-enabled supply chain. The model is shown schematically in Fig. 9.

- A company that plans to adopt Blockchain for the traceability of their products requests a track and trace service for their supply chain.

- The quantity of items is entered into a system that generates an MID (MOSChain Identity). The MIDs are produced through a mining process following a mining algorithm such as delegated PoS, delegated PoA, or PoW.

- The successful miner is rewarded with a MOHBS token.

- Two different types of tokens are introduced; MOS (smart payment currency for utilization in financial and 


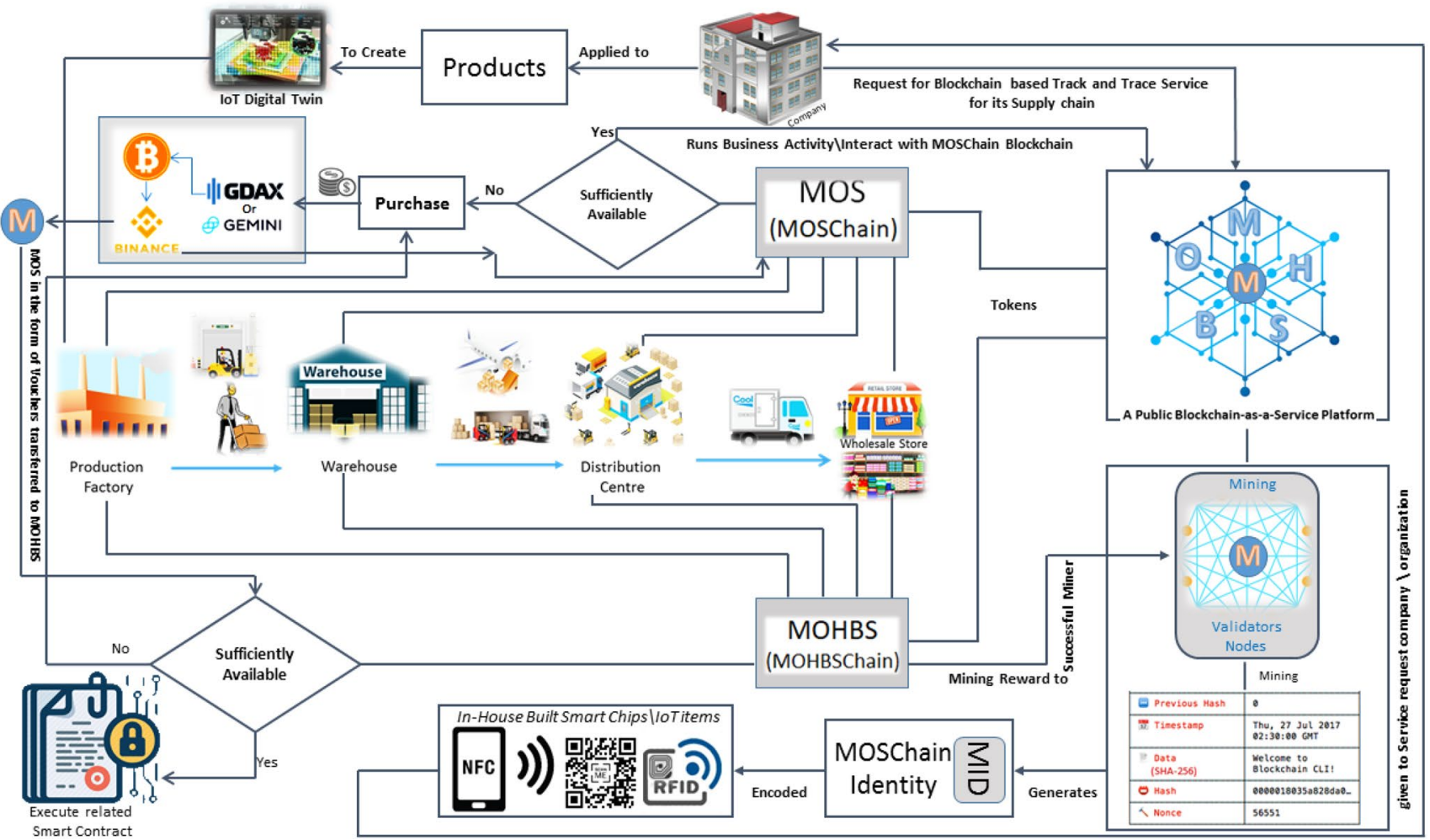

Fig. 9 Proposed Blockchain-enabled supply chain framework - MOHBSChain

business activities) and MOHBS (currency for the execution of smart contracts and for running the applications on the Blockchain).

- The MID is encoded to the in-house built IoT items (NFS chip, RFID trackers, QR codes, etc.) to track the item throughout their life cycle.

- The company uses these encoded IoT items on their products, as well as elevating them to the system's digital twin.

- As the products are moved to various departments (production factory, warehouse, distribution center, logistics and wholesale or retail stores, etc.), related records at the Blockchain service provider are updated.

- Programmed logic related to transaction and business agreements among the stakeholders are encoded in smart contracts which require MOHBS tokens for their execution.

- If MOHBS tokens are insufficient, they can be purchased in the form of MOS vouchers, further transferred to MOHBS tokens, since MOS is the smart currency of this Blockchain. FIAT currency is exchanged through GEMINI or GDAX into bitcoin that is further exchanged to MOS through Binance, for example.

- To run business activities or to interact with the MOSChain Blockchain, the smart cryptocurrency, MOS, is used. Any insufficiency is resolved through its purchase from Binance with the exchange of FIAT currency into Bitcoin and then to the MOS.

\section{Summary}

The supply chain is a complex system which comprises many components and stakeholders. Digital initiatives presenting a holistic and centralized approach have already played a role in improving the integration and standardization of its siloed processes: ordering, purchasing, manufacturing and logistics. GS1 has been instrumental in setting up standards for improving the visibility, efficiency and safety of the supply chain network in both physical or digital aspects. Traceability, which manages the process and product pedigree, ensures that the flow of goods is well monitored, effectively integrated, and efficiently recorded. Among others, data tampering, single point of failure with a centralized storage, dubious traceabilty information, distrust of authorities on data authenticity and lack of provenance are major issues in existing supply chain traceability systems. EPCIS-based systems have gone some way to resolving these issues, but much remains unsolved. Due to the promises which Blockchain makes in immutability, transparency, security, and fault tolerance, it is an appealing solution, at least for trust 
and traceability, and there are already many real use cases in the supply chain.

According to analysts at InnovatorâĂŹs Guide, there are around 800 startups around the globe working in the field of Blockchain. Even so, much groundwork is lacking before Blockchain can be widely adopted in applications, particularly in supply chain. A key issue at present is the orchestration of technical and non-technical constituents. There are many technical and non-technical challenges which must be addressed before the mass adoption of Blockchain possible; the major challenges are scalability and interoperability issues. The core component of a Blockchain technology is the Consensus algorithm that largely defines the performance of a Blockchain and its application suitability. Though a long list of consensus algorithms are available in the literature and each has its advantages and disadvantages, no one CA fits all situations, scenarios, and applications. PoW, PoS and BFT are widely used, either alone or in combination (as in SCP). For developing Blockchain-based applications, a development ecosystem comprises a Digital Ledger Technology (DLT) type, a choice of accessibility scope, a Blockchain platform, Consensus algorithm, and related tools such as Integrated Development Environment (IDE), testing tools and libraries, and programming language to define smart contracts and application logic, and storage applications. There are many options in these development layers, and a particular application will require a bespoke mix of these. Many large organizations have already taken steps toward implementation of Blockchain-enabled supply chain, with some encouraging results.

Open Access This article is licensed under a Creative Commons Attribution 4.0 International License, which permits use, sharing, adaptation, distribution and reproduction in any medium or format, as long as you give appropriate credit to the original author(s) and the source, provide a link to the Creative Commons licence, and indicate if changes were made. The images or other third party material in this article are included in the article's Creative Commons licence, unless indicated otherwise in a credit line to the material. If material is not included in the article's Creative Commons licence and your intended use is not permitted by statutory regulation or exceeds the permitted use, you will need to obtain permission directly from the copyright holder. To view a copy of this licence, visit http://creativecommons.org/licenses/by/4.0/.

\section{References}

1. Tseng, M.-L., Islam, M.S., Karia, N., Fauzi, F.A., Afrin, S.: A literature review on green supply chain management: trends and future challenges. Resources Conserv. Recycl. 141, 145-162 (2019)

2. Kelechi, G., Akujuobi, C. M., Sadiku, M. N., Chouikha, M., Alam, S.: Internet of things and blockchain integration: Use cases and implementation challenges, in: Business Information Systems Workshops: BIS 2019 International Workshops, Seville, Spain,
June 26-28, 2019, Revised Papers, volume 373, Springer Nature, p. 287(2020)

3. Bonazzoli, S., Borgianni, M., Falcone, C., Fioravanti, A., Longobardi, G., Lutri, S., Presti, L., Salerno, P., Tomasi, A., Ziantoni, F. et al.: Package delivery and reception with drones, (2020).US Patent 10,526,088

4. Angelini, F., Petrocelli, C., Catalano, M., Garabini, M., Grioli, G., Bicchi, A.: Softhandler: An integrated soft robotic system for the handling of heterogeneous objects. IEEE Robotics \& Automation Magazine (2020)

5. Hammoudeh, M., Ghafir, I., Bounceur, A., Rawlinson, T.: Continuous monitoring in mission-critical applications using the internet of things and blockchain, in: Proceedings of the 3rd International Conference on Future Networks and Distributed Systems, ICFNDS âĂŹ19, Association for Computing Machinery,New York, NY, USA, 2019. 10.1145/3341325.3342018

6. Rychtyckyj, N., Yang, H. H.: Handling rider service at autonomous vehicles, (2020).US Patent App. 16/023,874

7. Xie, R., Wang, Y., Tan, M., Zhu, W., Yang, Z., Wu, J., Jeon, G.: Ethereum-blockchain-based technology of decentralized smart contract certificate system. IEEE Internet Things Mag. 3, 44-50 (2020)

8. Wang, J., Han, K., Alexandridis, A., Chen, Z., Zilic, Z., Pang, Y., Jeon, G., Piccialli, F.: A blockchain-based ehealthcare system interoperating with wbans. Future Generation Comput. Syst. 110, 675-685 (2020)

9. Vidovič, E., Gajšek, B.: Analysing picking errors in vision picking systems. Logistics Sustain. Trans. 11, 90-100 (2020)

10. UK, G.: Welcome to gs1 uk, the global language of business - all you need to know about gs1 standards, Last accessed 06 January 2020.https://www.gs1uk.org/support-and-training/ourstandards

11. Singhal, B., Dhameja, G., Panda, P. S.: How blockchain works, in: Beginning Blockchain, Springer, pp. 31-148 (2018)

12. Kamble, S.S., Gunasekaran, A., Sharma, R.: Modeling the blockchain enabled traceability in agriculture supply chain. Int. J. Inform. Manag. 52, 101967 (2020)

13. Lin, Q., Wang, H., Pei, X., Wang, J.: Food safety traceability system based on blockchain and epcis. IEEE Access 7, 20698-20707 (2019)

14. Moin, S., Karim, A., Safdar, Z., Safdar, K., Ahmed, E., Imran, M.: Securing iots in distributed blockchain: Analysis, requirements and open issues. Future Gener. Comput. Syst. 100, 325-343 (2019)

15. Mudassir, M., Bennbaia, S., Unal, D., Hammoudeh, M.: Timeseries forecasting of bitcoin prices using high-dimensional features: a machine learning approach. Neural Computing and Applications 1-15 (2020)

16. Unal, D., Hammoudeh, M., Kiraz, M. S.: Policy specification and verification for blockchain and smart contracts in $5 \mathrm{~g}$ networks, ICT Express 6 (2020) 43 - 47. http://www.sciencedir ect.com/science/article/pii/S240595951930181X. https://doi. org/10.1016/j.icte.2019.07.002

17. Jangirala, S., Das, A.K., Vasilakos, A.V.: Designing secure lightweight blockchain-enabled rfid-based authentication protocol for supply chains in $5 \mathrm{~g}$ mobile edge computing environment. IEEE Transactions on Industrial Informatics (2019)

18. Kravitz, D. W.: Transaction immutability and reputation traceability: Blockchain as a platform for access controlled iot and human interactivity, in: 2017 15th Annual Conference on Privacy, Security and Trust (PST), IEEE, pp. 3-309 (2017)

19. Koirala, R. C., Dahal, K., Matalonga, S.: Supply chain using smart contract: a blockchain enabled model with traceability and ownership management, in: 2019 9th International Conference on Cloud 
Computing, Data Science \& Engineering (Confluence), IEEE, pp. 538-544 (2019)

20. Imeri, A., Khadraoui, D.: The security and traceability of shared information in the process of transportation of dangerous goods, in: 2018 9th IFIP International Conference on New Technologies, Mobility and Security (NTMS), IEEE, pp. 1-5 , (2018)

21. Yapa, I., Heanthenna, S., Bandara, N., Prasad, I., Mallawarachchi, Y., Decentralized ledger for land and property transactions in sri lanka acresense, in, : IEEE region 10 humanitarian technology conference (R10-HTC). IEEE 2018, 1-6 (2018)

22. Shahid, M. R., Mahmood, S., Hafeez, S., Zahid, B., Jabbar, S., Ashraf, R.: Blockchain based share economy trust point: Case study based validation, in: Proceedings of the 3rd International Conference on Future Networks and Distributed Systems, ACM , p. $41,(2019)$

23. Wang, S., Li, D., Zhang, Y., Chen, J.: Smart contract-based product traceability system in the supply chain scenario. IEEE Access 7, 115122-115133 (2019)

24. Liu, D., Alahmadi, A., Ni, J., Lin, X., Shen, X.: Anonymous reputation system for iiot-enabled retail marketing atop pos blockchain. IEEE Trans. Ind. Inform. 15, 3527-3537 (2019)

25. Ferrag, M.A., Maglaras, L.: Deepcoin: A novel deep learning and blockchain-based energy exchange framework for smart grids. IEEE Transactions on Engineering Management (2019)

26. Gul, M. j. j., Paul, A., Ahmad, A., Khan, M., Jeon, G.: Smart contract's interface for user centric business model in blockchain, in: Proceedings of the 34th ACM/SIGAPP Symposium on Applied Computing, pp. 709-714 (2019),

27. Jogunola, O., Hammoudeh, M., Adebisi, B., Anoh, K., Demonstrating blockchain-enabled peer-to-peer energy trading and sharing, in, : IEEE Canadian conference of electrical and computer engineering (CCECE). IEEE 2019, 1-4 (2019)

28. Habib, M. A., Sardar, M. B., Jabbar, S., Faisal, C. N., Mahmood, N., Ahmad, M.: Blockchain-based supply chain for the automation of transaction process: Case study based validation, in: 2020 International Conference on Engineering and Emerging Technologies (ICEET), IEEE, pp. 1-7 (2020)

29. Hammoudeh, M., Ghafir, I., Bounceur, A., Rawlinson, T.: Continuous monitoring in mission-critical applications using the internet of things and blockchain, in: Proceedings of the 3rd International Conference on Future Networks and Distributed Systems , pp. 1-5 (2019)

30. Shanaev, S., Shuraeva, A., Vasenin, M., Kuznetsov, M.: Cryptocurrency value and $51 \%$ attacks: evidence from event studies. J. Altern. Invest. 22, 65-77 (2019)

31. Boireau, O.: Securing the blockchain against hackers. Netw. Secur. 2018, 8-11 (2018)

32. Tariq, N., Asim, M., Al-Obeidat, F., Zubair Farooqi, M., Baker, T., Hammoudeh, M., Ghafir, I.: The security of big data in fogenabled iot applications including blockchain: a survey, Sensors 19, $1788(2019)$

33. GUTIERREZ, C.: Blockchain at walmart: Tracking food from farm to fork, Last accessed 12 January 2020. https://www.altoros.com/ blog/blockchain-at-walmart-tracking-food-from-farm-to-fork/

34. Kamath, R.: Food traceability on blockchain: WalmartâĂŹs pork and mango pilots with ibm. J. Br. Blockchain Assoc. 1, 3712 (2018)

35. Foundation, T. L.: Case study:how walmart brought unprecedented transparency to the food supply chain with hyperledger fabric, https://www.hyperledger.org/resources/publications/ walmart-case-study (2020)

36. Company, A. D. N. O.: Adnoc announces groundbreaking blockchain application for the oil and gas production value chain using ibm blockchain - abu dhabi national oil company, Last accessed 03 January 2020.https://www.adnoc.ae/en/news-and-media/press -releases/2018/adnoc-announces-groundbreaking-blockchain -application

37. De Angelis, S., Aniello, L., Baldoni, R., Lombardi, F., Margheri, A., Sassone, V.: Pbft vs proof-of-authority: applying the cap theorem to permissioned blockchain (2018)

38. Aitken, R.: Ibm \& walmart launching blockchain food safety alliance in china with fortune 500's jd.com - forbes now, Last accessed 03 January 2020. https://www.forbes.com/sites/roger aitken/2017/12/14/ibm-walmart-launching-blockchain-food-safet y-alliance-in-china-with-fortune-500s-jd-com/\#31971a7e7d9c

39. L. D. Company, Ldc and partners use blockchain to achieve full traceability, Last accessed 03 January 2020. https://www.ldc.com/ global/en/our-stories/in-field/ldc-and-partners-use-blockchain -achieve-full-traceability/

40. Jarlgaard, M.: About - martine jarlgaard logo image, Last accessed 03 January 2020. https://martinejarlgaard.com/About

41. OriginTrail, Utilizing smart sensors to prevent wine fraud âĂ ̌ origintrailâǍŽs pilot with tagitsmar - medium, Last accessed 03 January 2020. https://medium.com/origintrail/utilizing-smart -sensors-to-prevent-wine-fraud-origintrails-pilot-with-tagit smart-1949dc62113f

42. TRUSTNODES, World's largest freight company to use blockchain tech for asset management - trustnodes, Last accessed 03 January 2020. https://www.trustnodes.com/2016/09/06/world s-largest-freight-company-use-blockchain-tech-asset-manag ement

43. Allison, I.: Blockchain traceable coffee: bext 360 partners in africa, europe and north america - international business times, October 31, 2017.https://www.ibtimes.co.uk/blockchain-traceable-coffe e-bext360-partners-africa-europe-north-america-1645350

44. BanQu, Case study: Blockchain for supply chain transparency - banqu supply chain management - cost savings, brand enhancement, long-term success - banqu - dignity through identity, Last accessed 03 January 2020. https://banqu.co/case-study/

45. Kemp, L.: Walmart and ibm are partnering to put chinese pork on a blockchain, Last accessed 12 January 2020.https://fortu ne.com/2016/10/19/walmart-ibm-blockchain-china-pork/

46. Tom Wilson, E. A.: Chickens and eggs: Retailer carrefour adopts blockchain to track fresh produce, Last accessed 12 January 2020. https://www.reuters.com/article/us-carrefour-blockchain-ibm/ chickens-and-eggs-retailer-carrefour-adopts-blockchain-to-track -fresh-produce-idUSKCN1MI162

47. Brody, P.: How blockchain is revolutionizing supply chain management. Digitalist Magazine 1-7 (2017)

48. Scott, T.: Blockchain - tradelens: How ibm and maersk are sharing blockchain to build a global trade platform, Last accessed 03 January 2020.https://www.ibm.com/blogs/think/2018/11/ tradelens-how-ibm-and-maersk-are-sharing-blockchain-to-build -a-global-trade-platform/

49. Meat, M., Australia, L.: Evaluation of blockchain technology, Last accessed 03 January 2020.https://www.mla.com.au/research-anddevelopment/search-rd-reports/final-report-details/Evaluation-ofBlockchain-Technology/4120\#

50. Lau, H.: How blockchain technology can reassure food consumers, Last accessed 13 January 2020. https://troymedia.com/business/ bixs-will-utilize-blockchain-technology/

51. Insights, S.: 8 blockchain startups disrupting the supply chain industry, Last accessed 12 January 2020. https://www.startus-insig hts.com/innovators-guide/8-blockchain-startups-disrupting-thesupply-chain-industry/

52. Queiroz, M.M., Wamba, S.F.: Blockchain adoption challenges in supply chain: an empirical investigation of the main drivers in india and the usa. Int. J. Inform. Manag. 46, 70-82 (2019)

53. Deshpande, A., Stewart, K., Lepetit, L., Gunashekar, S.: Distributed ledger technologies/blockchain: Challenges, opportunities 
and the prospects for standards, Overview report The British Standards Institution (BSI) 1-34 (2017)

54. Kim, S., Kwon, Y., Cho, S.: A survey of scalability solutions on blockchain, in: 2018 International Conference on Information and Communication Technology Convergence (ICTC), IEEE, pp. 1204-1207 (2018)

55. Hazari, S.S., Mahmoud, Q.H., A parallel proof of work to improve transaction speed and scalability in blockchain systems, in, : IEEE 9th annual computing and communication workshop and conference (CCWC). IEEE 2019, 0916-0921 (2019)

56. Chen, H., Wang, Y.: Sschain: A full sharding protocol for public blockchain without data migration overhead. Pervasive Mobile Comput. 59, 101055 (2019)

57. Lee, S., Kim, H.: On the robustness of lightning network in bitcoin. Pervasive Mob. Comput. 61, 101108 (2020)

58. Bez, M., Fornari, G., Vardanega, T.: The scalability challenge of ethereum: An initial quantitative analysis, in: 2019 IEEE International Conference on Service-Oriented System Engineering (SOSE), IEEE, pp. 167-176 (2019)

59. Xie, J., Yu, F.R., Huang, T., Xie, R., Liu, J., Liu, Y.: A survey on the scalability of blockchain systems. IEEE Netw. 33, 166-173 (2019)

60. Cao, B., Zhang, Z., Feng, D., Zhang, S., Zhang, L., Peng, M., Li, Y.: Performance analysis and comparison of pow, pos and dag based blockchains, Digital Communications and Networks (2020)

61. Koens, T., Poll, E.: Assessing interoperability solutions for distributed ledgers. Pervasive Mob. Comput. 59, 101079 (2019)

62. Johnson, S., Robinson, P., Brainard, J.: Sidechains and interoperability, arXiv preprint arXiv:1903.04077 (2019)

63. Deng, L., Chen, H., Zeng, J., Zhang, L.-J.: Research on crosschain technology based on sidechain and hash-locking, in: International Conference on Edge Computing, Springer, pp. 144-151 (2018),

64. Project, I.: Interledger protocol v4, Last accessed 06 January 2020.https://interledger.org/rfcs/0027-interledger-protocol-4/

65. Liu, S., Viotti, P., Cachin, C., Quéma, V., Vukolić, M.: $\{X F T\}$ : Practical fault tolerance beyond crashes, in: 12th \{USENIX\} Symposium on Operating Systems Design and Implementation (\{OSDI\} 16), pp. 485-500 (2016)

66. Miller, A., Xia, Y., Croman, K., Shi, E., Song, D.: The honey badger of bft protocols, in: Proceedings of the 2016 ACM SIGSAC Conference on Computer and Communications Security, ACM, pp. 31-42 (2016)

67. Yang, Y.: Linbft: Linear-communication byzantine fault tolerance for public blockchains, arXiv preprint arXiv:1807.01829 (2018)

68. Crain, T., Gramoli, V., Larrea, M., Raynal, M.: Dbft: Efficient byzantine consensus with a weak coordinator and its application to consortium blockchains, arXiv preprint arXiv:1702.03068 (2017)

69. Gągol, A., Leśniak, D., Straszak, D., Świętek, M.: Aleph: Efficient atomic broadcast in asynchronous networks with byzantine nodes, in: Proceedings of the 1st ACM Conference on Advances in Financial Technologies, pp. 214-228 (2019)

70. Ongaro, D.: Consensus: Bridging theory and practice, Ph.D. thesis, Stanford University, (2014)

71. Eyal, I., Gencer, A. E., Sirer, E. G., Van Renesse, R.: Bitcoin-ng: A scalable blockchain protocol, in: 13th \{USENIX\} Symposium on Networked Systems Design and Implementation (\{NSDI\} 16), pp. 45-59 (2016)

72. Sylim, P., Liu, F., Marcelo, A., Fontelo, P.: Blockchain technology for detecting falsified and substandard drugs in distribution: pharmaceutical supply chain intervention. JMIR Res. Protoc. 7, e10163 (2018)

73. Mao, D., Hao, Z., Wang, F., Li, H.: Innovative blockchain-based approach for sustainable and credible environment in food trade: a case study in shandong province, china. Sustainability 10, 3149 (2018)
74. Huang, Y., Wu, J., Long, C.: Drugledger: A practical blockchain system for drug traceability and regulation, in: 2018 IEEE International Conference on Internet of Things (iThings) and IEEE Green Computing and Communications (GreenCom) and IEEE Cyber, Physical and Social Computing (CPSCom) and IEEE Smart Data (SmartData), IEEE, pp. 1137-1144 (2018)

75. Kuhn, M., Giang, H., Otten, H., Franke, J.: Blockchain enabled traceability-securing process quality in manufacturing chains in the age of autonomous driving, in: 2018 IEEE International Conference on Technology Management, Operations and Decisions (ICTMOD), IEEE, pp. 131-136 (2018)

76. Westerkamp, M., Victor, F., Küpper, A.: Blockchain-based supply chain traceability: Token recipes model manufacturing processes, in: 2018 IEEE International Conference on Internet of Things (iThings) and IEEE Green Computing and Communications (GreenCom) and IEEE Cyber, Physical and Social Computing (CPSCom) and IEEE Smart Data (SmartData), IEEE, pp. 15951602 (2018)

77. Salah, K., Nizamuddin, N., Jayaraman, R., Omar, M.: Blockchainbased soybean traceability in agricultural supply chain. IEEE Access 7, 73295-73305 (2019)

78. Baralla, G., Pinna, A., Corrias, G., Ensure traceability in european food supply chain by using a blockchain system, in, : IEEE/ACM 2nd international workshop on emerging trends in software engineering for blockchain (WETSEB). IEEE 2019, 40-47 (2019)

79. Helo, P., Shamsuzzoha, A.: Real-time supply chaina blockchain architecture for project deliveries. Robot. Comput. Integrated Manuf. 63, 101909 (2020)

80. Knirsch, F., Unterweger, A., Engel, D.: Implementing a blockchain from scratch: why, how, and what we learned. EURASIP J. Inform. Secur. 2019, 2 (2019)

81. Xu, X., Lu, Q., Liu, Y., Zhu, L., Yao, H., Vasilakos, A.V.: Designing blockchain-based applications a case study for imported product traceability. Future Gener. Comput. Syst. 92, 399-406 (2019)

82. Cuomo, G. A., Dillenberger, D. N., Heath III, F. F., Liu, R., Vaculin, R.: Automatic generating analytics from blockchain data, (2019)

83. Dillenberger, D., Novotny, P., Zhang, Q., Jayachandran, P., Gupta, H., Hans, S., Verma, D., Chakraborty, S., Thomas, J., Walli, M., et al.: Blockchain analytics and artificial intelligence. IBM J. Res. Dev. 63, 1-5 (2019)

84. Farhan, M., Jabbar, S., Aslam, M., Ahmad, A., Iqbal, M.M., Khan, M., Maria, M.-E.A.: A real-time data mining approach for interaction analytics assessment: Iot based student interaction framework. Int. J. Parallel Program. 46, 886-903 (2018)

85. Tateishi, T., Yoshihama, S., Sato, N., Saito, S.: Automatic smart contract generation using controlled natural language and template. IBM J. Res. Dev. 63, 1-6 (2019)

86. Wang, S., Ouyang, L., Yuan, Y., Ni, X., Han, X., Wang, F.-Y.: Blockchain-enabled smart contracts: architecture, applications, and future trends. IEEE Trans. Syst. Man Cybern 49, 2266-2277 (2019)

87. Rouhani, S., Deters, R.: Security, performance, and applications of smart contracts: a systematic survey. IEEE Access 7, 5075950779 (2019)

88. Katina, P.F., Keating, C.B., Sisti, J.A., Gheorghe, A.V.: Blockchain governance. Int. J. Crit. Infrastruct. 15, 121-135 (2019)

89. Rikken, O., Janssen, M., Kwee, Z.: Governance challenges of blockchain and decentralized autonomous organizations. Information Polity 1-21 (2019)

90. Guo, L., Xie, H., Li, Y.: Data encryption based blockchain and privacy preserving mechanisms towards big data. J. Vis. Commun. Image Representation 102741, (2019)

91. Perrons, R.K., Cosby, T.: Applying blockchain in the geoenergy domain: the road to interoperability and standards. Appl. Energy 262, 114545 (2020) 
92. Zhou, Q., Huang, H., Zheng, Z., Bian, J.: Solutions to scalability of blockchain: a survey. IEEE Access 8, 16440-16455 (2020)

93. Shawn Muma, R. S., David Kappos, The right to be forgotten meets the immutable - a practical guide to gdpr-compliant blockchain solutions, Last accessed 12 February 2020. https://www. cravath.com/files/Uploads/Documents/Publications/

94. Humbeeck, A.V.: The blockchain-gdpr paradox. J. Data Protection Privacy 2, 208-212 (2019)
Publisher's Note Springer Nature remains neutral with regard to jurisdictional claims in published maps and institutional affiliations. 